\title{
A Novel Selected Subcarrier Method for Digital Video Broadcasting
}

\author{
Chandra kala. K, Jaya Kumari. J, Sreeja. T. K
}

\begin{abstract}
In Digital Video broadcasting there are two types of information namely global and local content. Broadcasting both information using multifrequency network requires large spectrum. To overcome that single frequency network is incorporated.Inorder to transmit both the information in a single frequency network, a novel technique, namely selected subcarrier method of Digital Video Broadcasting is proposed. This technique is well suited in a scenario where the transmission of two different information is targeted over the entire globe at the same time. This methodology proficiently handles the concept of Orthogonality principles among the OFDM subcarrier. In this technique certain dedicated subcarrier is used for transmission of Local content in the network.Also, wavelet-based OFDM has been proposed, which outperforms the existing Fourier based OFDM
\end{abstract}

Keywords: DVB, Global content, Local content, Orthogonality, OFDM, Subcarriers

\section{INTRODUCTION}

Digital television is a technology of television broadcasting to customers in digital format. This replaces the analog television successfully with high-quality digital service. It offers high definition TV signals. In addition, it uses the spectrum more efficiently. All through the globe, there are different standards for digital terrestrial television broadcasting. One among that is the DVB standard. Digital broadcasting through DVB system targets transmission of two different types of information based on the network coverage. Some information is the primary contents that are aimed over entire region is the global content. Specifically some information is targeted to certain region known as local information. Transmitting Global and Local Content using different transmitters of different frequency consumes large spectrum. Instead, the proposed technique utilizes the features of single frequency network incorporated with OFDM. The subcarriers generated by the IFFT block of OFDM are orthogonal to each other [1]. Thereby the subdivided low rate information streams modulated with these carriers are uninterfered with each other. Thus the frequency pre-owned by a sub-stream at the transmitter must be used at the receiver to recover the information. This concept is well suited for the proposed work.

Revised Manuscript Received on October 30, 2019.

* Correspondence Author

Chandra Kala.K*, ECE, Noorul Islam Centre for Higher Education, Tamil Nadu,India. Email:chandrakalakigin@gmail.com

Jaya Kumari J, ECE, Mar Baselios College of Engineering and Technology, Kerala, India. Email: jayakumarij72@gmail.com

Sreeja.T.K, Nano Technology, Noorul Islam Centre for Higher Education, Tamil Nadu,India. Email: sreejaeng07@gmail.com Sciences Publication (BEIESP). This is an open access article under the CC BY-NC-ND license (http://creativecommons.org/licenses/by-nc-nd/4.0/)
(C) The Authors. Published by Blue Eyes Intelligence Engineering and

Among the data, subcarriers certain dedicated subcarriers are allotted for local content and the remaining are assigned for global contents. The different transmitter in the local service region reserves a different set of subcarriers to broadcast local content within same OFDM symbol. Since the subcarriers are orthogonal there will be no interference occurs. This is the added advantage in local service transmission in SFN network by this technique. A special reuse pattern is also applicable here to reuse frequencies of the subcarrier separated by particular distances. As the OFDM symbols are non-hierarchically modulated, both global and local service provides same coverage and performance. The coverage area and performance of the global service is not affected by the local service. Cyclic prefix append at the start of the OFDM symbol avoids intersymbol interference [1].

At present digital video broadcasting with OFDM is implemented using Fast Fourier transform. Transmission of signals in wireless channels results in multiple copies of the signal at the receiver and this leads to interference. In OFDM, for combating with inter symbol inference; a guard interval known as the cyclic prefix is inserted between two active OFDM symbols. Even though this is an effective solution for interference, it consumes $20 \%$ to $40 \%$ of bandwidth available. To solve this problem wavelet OFDM has been proposed. As the wavelet is more resistant to interference, it does not require cyclic prefix, which results in saving of bandwidth. It also improves the bit error rate performance of both the streams.

\section{RELATED WORK}

Presently transmission of local and global content in Single Frequency Network is done using hierarchical modulation technique [2], [3], [4], where local, global services are separately encoded, modulated, and QAM mapped to get the complex hierarchically modulated signal [5]. In this method, the local content is transmitted as Low Priority Stream (LP) and global contents as High Priority Stream (HP). Both local and global contents are embedded in single constellation. This is achieved by selection of appropriate modulation schemes for HP and LP. Fig.1 clearly depicts the resulting lower-order 16HQAM signal constellation. The first two bit represents the HP or global content that is the same for all the signal points within the quadrant. The remaining bits changes within the quadrant represent the LP local content. In general QPSK modulation is preferred for HP contents. However to achieve $2^{\mathrm{n}}$ HQAM constellation, LP content uses $2^{\mathrm{n}-2}$ QAM scheme. The bit error rate of the LP stream is quite higher compared to the HP stream [6], [7]. There is a decoding complexity in case of hierarchical modulation schemes [8] The decoded signal provides more bit error rate than the theoretical non-hierarchical modulated signal. 


\section{A Novel Selected Subcarrier Method for Digital Video Broadcasting}

Similarly the case is same for local information decoded in different region. To overcome this limitation a new technique is proposed in this paper, which takes advantage of the Orthogonality Property of the OFDM subcarriers. The local service can be provided over the entire network with same coverage as the global services. Also since the subcarriers are orthogonal, there is no interference between the local services. The decoding complexity loaded in the Existing system is overcome in the proposed technique.

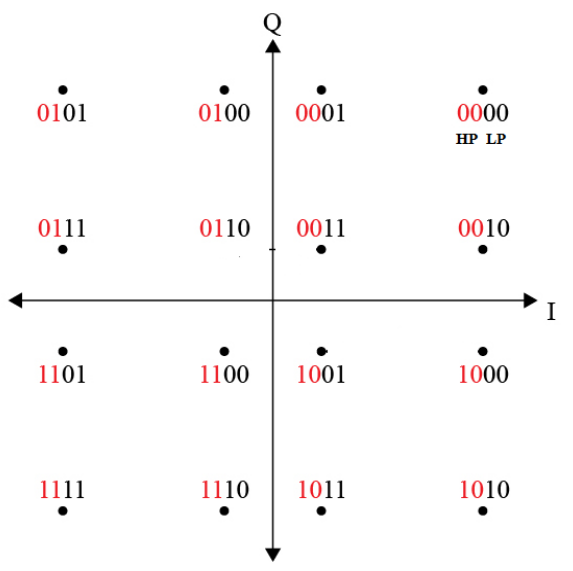

Fig 1: Constellation Diagram of 16HQAM signal transmission scheme .recent years, the application of wavelet OFDM in the broadcasting system is keeping on increasing .many areas like hybrid power line communication[9], visible light communication[9],5G communication[10],[11], cognitive radio[12], Multicarrier CDMA[13], UWB system, cognitive UWB[14], and satellite communication uses wavelet OFDM. In conventional FFT there is a requirement cyclic prefix insertion to reduce the intersymbol interference, which consumes large bandwidth. [15-18].To overcome this, the wavelet OFDM is introduced. This saves bandwidth of nearly $20 \%$ to $40 \%$.

\section{PROPOSED SYSTEM}

The Proposed selected subcarrier method of DVB is given in fig 2. To improve the performance of the wireless system, BICM is used which is the combination of a serial concatenation of two coders and an interleaver followed by the modulator. In this technique, the global and local contents are separately encoded and modulated. The modulation scheme is selected based on the requirement. Unlike Hierarchical modulation, any scheme of mapping can be used.QAM and QPSK schemes are generally preferred.QAM of order 16, 64 and 256 are accepted for the DVB system. In this system, there is no compulsion for the global content to be modulated with QPSK mapping scheme. Since both global and local content is non-hierarchically modulated, there is no degradation in system performance. In this method, the allocation of a subcarrier for transmission of local content is done before the IFFT operation. The modulated complex signal is broken to sub-streams of length $\mathrm{N}$ form blocks of the subchannel. In the $\mathrm{N}$ length block, at the dedicated index, the local content is placed among the useful data carrier. At the receiver, the respective carrier index is demodulated with the carrier of the same frequency to get the local and global content successfully.
Wavelet OFDM is the challenging technique in multicarrier

Let $X_{I}(k)$, be the input modulated signal consist of both global and local content i.e., $X_{I}(k) \in\left\{X_{g}(k), X_{l}(k)\right\}$.where $X_{g}$ be the modulated complex global content, $X_{l}$ be the modulated local content and $X_{I}(k)$ be the frequency domain input to the IFFT block. In the IFFT operation, the signal is multiplied with sinusoids of different frequencies thereby breaks the signal to different frequency bins. This is the concept that supports the transmission of local information along with global information in coded OFDM based single frequency network. The input complex signal of the IFFT includes both local and global content located based on the carrier index selected for transmission of respective contents. The IFFT operation is represented in matrix form for a subcarrier of $\mathrm{N}=8$ is given in equation 1

$\left[\begin{array}{llllllll}w_{8}^{0} & w_{8}^{0} & w_{8}^{0} & w_{8}^{0} & w_{8}^{0} & w_{8}^{0} & w_{8}^{0} & w_{8}^{0} \\ w_{8}^{0} & w_{8}^{1} & w_{8}^{2} & w_{8}^{3} & w_{8}^{4} & w_{8}^{5} & w_{8}^{6} & w_{8}^{7} \\ w_{8}^{0} & w_{8}^{2} & w_{8}^{4} & w_{8}^{6} & w_{8}^{0} & w_{8}^{2} & w_{8}^{4} & w_{8}^{6} \\ w_{8}^{0} & w_{8}^{3} & w_{8}^{6} & w_{8}^{1} & w_{8}^{4} & w_{8}^{7} & w_{8}^{2} & w_{8}^{5} \\ w_{8}^{0} & w_{8}^{4} & w_{8}^{0} & w_{8}^{4} & w_{8}^{0} & w_{8}^{4} & w_{8}^{0} & w_{8}^{4} \\ w_{8}^{0} & w_{8}^{5} & w_{8}^{2} & w_{8}^{7} & w_{8}^{4} & w_{8}^{1} & w_{8}^{6} & w_{8}^{3} \\ w_{8}^{0} & w_{8}^{6} & w_{8}^{4} & w_{8}^{2} & w_{8}^{0} & w_{8}^{6} & w_{8}^{4} & w_{8}^{2} \\ w_{8}^{0} & w_{8}^{7} & w_{8}^{6} & w_{8}^{5} & w_{8}^{4} & w_{8}^{3} & w_{8}^{2} & w_{8}^{1}\end{array}\right] \cdot\left[\begin{array}{c}X(0) \\ X_{g}(1) \\ X_{l}(2) \\ X_{g}(3) \\ X_{l}(4) \\ X_{g}(5) \\ X_{l}(6) \\ X_{g}(7)\end{array}\right]=\left[\begin{array}{c}x(0) \\ x_{g}(1) \\ x_{l}(2) \\ x_{g}(3) \\ x_{l}(4) \\ x_{g}(5) \\ x_{l}(6) \\ x_{g}(7)\end{array}\right]$

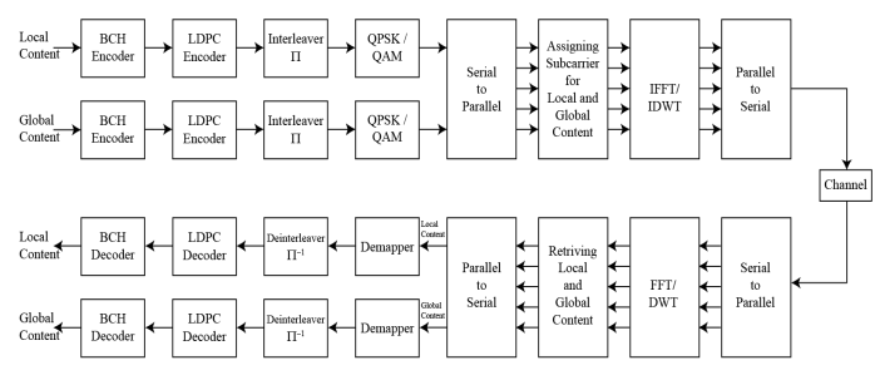

Fig 2.Proposed DVB system model using the selected subcarrier method

Among the $\mathrm{N}$ subcarrier, certain dedicated carriers are used to transmit the local contents, which are optional and vary for the different SFN transmitter. For understanding, in equation 1, index 2, 4, 6 is considered for local content. There are DC carrier, pilot carriers for channel estimation and equalization, unused carrier and data carriers are available among the OFDM subcarriers. The output of the IFFT operator is given as in equation 2

$$
x(n)=\sum_{k=0}^{N-1} X_{I}(k) \cdot W_{N}^{n k}, n=0,1,2, \ldots N-1
$$

Where $x(n)=\left\{x_{g}(n), x_{l}(n)\right\}, x_{g}$ represent the global content and $x_{l}$ denote the local content. The subchannel of OFDM prevails certain characteristics as stated below

1. The sub-channels divided are of data rate much less the data rate of the entire channel and its respective bandwidth is lesser than the entire bandwidth. 
2. The number of the subchannel is such that each bandwidth must be small. So that the frequency response of each sub-channels frequency range is almost small. This reduces intersymbol interference.

3. The sub-channels don't need to be contiguous therefore; a large block of continuous spectrum is not required for transmitting signal.

4. Each subchannel can use different modulation formats. This selection of modulation scheme depends on the channel quality and system requirement.

\section{ORTHOGONALITY OF THE SUBCARRIERS}

The OFDM receiver receives the signal $Y_{l}[n]$ as the input to the FFT operation. The $\mathrm{K}^{\text {th }}$ subcarrier frequency components of the $l^{\text {th }}$ transmitted symbol are $Y_{l}(k)$.Then the received signal can be written as in equation 3

$$
Y_{l}(k)=\sum_{n=0}^{N-1} Y_{l}[n] e^{-\frac{j 2 \pi k n}{N}}
$$

(3)

Using equation 2 in 3

$$
\begin{aligned}
& =\sum_{n=0}^{N-1}\left\{\frac{1}{N} \sum_{i=1}^{N-1} X_{l}[i] \mathrm{e}^{\frac{\mathrm{j} 2 \pi \mathrm{in}}{N}}\right\} e^{-j 2 \pi k n} \\
& =\frac{1}{N} \sum_{n=0}^{N-1} \sum_{i=1}^{N-1} X_{l}[\mathrm{i}] \exp \left(\frac{\mathrm{j} 2 \pi(\mathrm{i}-\mathrm{k}) n}{N}\right) \\
& =X_{l}[k] \quad\{\mathrm{i}=\mathrm{k}\}
\end{aligned}
$$

The above equation shows the orthogonality is maintained at the receiver, The OFDM symbol modulated with $i^{\text {th }}$ carrier demodulated with $i^{\text {th }}$ subcarrier produce nonzero value. Therefore, the respective subcarrier frequency modulates the local and global content is used to demodulate the signal at the receiver. Therefore, the local content and global content remain uninterfered as illustrated in equation 5 and the spectrum in Figure 3.

$$
Y_{I}[n]= \begin{cases}Y_{g}[n], & \mathrm{K}_{\mathrm{g}}=0,1,2, \ldots . . N_{g}-1 \\ Y_{l}[n], & \mathrm{K}_{\mathrm{l}}=0,1,2, \ldots . . N_{l}-1\end{cases}
$$

Where, $N=N_{g}+N_{l}+N_{o} . N_{g}$ denotes the number of the carrier for global content and $N_{l}$, the number of carriers for local content. $N_{o}$ denotes the carriers other than the data carrier used in OFDM symbol.

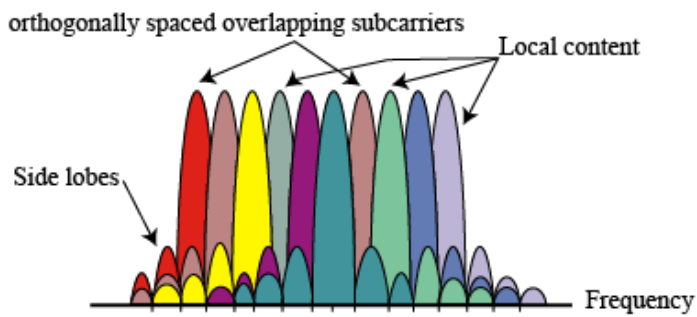

Fig 3.The spectrum of orthogonal carriers of local and global content

\section{SIMUATION RESULTS}

\subsection{Analysis of Proposed System with FFT based and wavelet-based OFDM:}

The analysis of the performance of the proposed system with FFT based and Wavelet-based OFDM is shown in fig. 4 and 5.It is noted from the figure that the wavelet-based system provides good performance compared to FFT based system.

Table 1.Simulation Parameters for Analysi
\begin{tabular}{|l|l|}
\hline Specification & Parameters \\
\hline Outer Coder & BCH \\
\hline Inner Coder & LDPC \\
\hline Code Rate & $7 / 15$ \\
\hline Transform Size & $2 \mathrm{k}$ \\
\hline Wavelet & Daubechies(Db4) \\
\hline \multicolumn{2}{|c|}{ Modulation Schemes } \\
\hline Case I & $\begin{array}{l}\text { Global Content:QPSK } \\
\text { Local Content:QPSK }\end{array}$ \\
\hline Case II & $\begin{array}{l}\text { Global Content:QPSK } \\
\text { Local Content:QAM }\end{array}$ \\
\hline
\end{tabular}

Case I: In the case shown in Fig 4, when global and local content is QPSK modulated. To achieve an error rate of $10^{-4}$, the SNR requirement of FFT based system is 3dB greater than the wavelet-based system. The BER of both the streams shows the same variation since the local content and global content are transmitted with same energy level. This does not affect the transmission of information because only the value of the complex signal is the same but the subcarrier carries both the information are orthogonal.

Case II: The analysis is extended to another case where the local content is QAM modulated and global content is QPSK modulated. The Performance shown in fig.5 illustrates that the local content shows a higher error rate than the global content. This is because when modulation order increases, the data rate increases with sacrifice in error rate. The situation can be reversed by choosing the QAM for global content and QPSK for local content. The behavior of the system depends on the modulation scheme and power of the signal. Even with this case, the wavelet OFDM outperforms the FFT based system. To achieve $10^{-4}$ error rate, the local content of the FFT based system requires $13 \mathrm{~dB}$ SNR and $11 \mathrm{~dB}$ for wavelet-based system There is a difference in SNR of 2dB.On the other hand, the global content of FFT based system requires $18 \mathrm{~dB}$ SNR and for the wavelet-based system, it is $3 \mathrm{~dB}$ lesser than that. The simulation parameter for analysis is shown in table 1 . The numerical value of the BER for Fourier based and wavelet-based systems for two cases are given in table 2 . 
A Novel Selected Subcarrier Method for Digital Video Broadcasting

Table 2.BER of Selected Subcarrier Method for FFT and Wavelet-based System

\begin{tabular}{|c|c|c|c|c|c|c|c|c|}
\hline \multirow{4}{*}{ SNR } & \multicolumn{8}{|c|}{ Bit Error Rate } \\
\hline & \multicolumn{4}{|c|}{ Global Content-QPSK; Local Content-QPSK } & \multicolumn{4}{|c|}{ Global Content-QPSK; Local Content-QAM } \\
\hline & \multicolumn{2}{|c|}{ FFT Based OFDM } & \multicolumn{2}{|c|}{ Wavelet OFDM } & \multicolumn{2}{|c|}{ FFT Based OFDM } & \multicolumn{2}{|c|}{ Wavelet OFDM } \\
\hline & Global & Local & Global & Local & Global & Local & Global & Local \\
\hline 1 & .1501 & .1529 & $\begin{array}{l}.0826 \\
\end{array}$ & .0803 & .1752 & .3013 & .0968 & .2460 \\
\hline 2 & .1318 & .1420 & .0576 & .0617 & .1433 & .2864 & .0662 & .2276 \\
\hline 3 & .1108 & .1064 & .0365 & .0375 & .1165 & .2686 & .0426 & 2005 \\
\hline 4 & .0825 & .0738 & .0240 & .0255 & .0995 & .2411 & .0281 & .1739 \\
\hline 5 & .0552 & .0516 & .0125 & .0120 & .0709 & .2214 & .0179 & .1478 \\
\hline 6 & .0368 & .0388 & .0048 & .0055 & .0428 & .2025 & .0081 & .1176 \\
\hline 7 & .0227 & .0220 & .0018 & .0025 & .0283 & .1750 & .0040 & .0924 \\
\hline 8 & .0136 & .0122 & .0008 & .0005 & .0139 & .1457 & .0011 & .0668 \\
\hline 9 & .0053 & .0065 & .0001 & .0004 & .0090 & .1197 & .0005 & .0483 \\
\hline 10 & .0024 & .0021 & 0 & .0001 & .0051 & .0882 & .0003 & .0281 \\
\hline 11 & .0009 & .0008 & 0 & 0 & .0015 & .0683 & .0002 & .0194 \\
\hline 12 & .0001 & .0002 & 0 & 0 & .0007 & .0492 & 0 & .0096 \\
\hline 13 & 0 & 0 & 0 & 0 & .0001 & .0296 & 0 & .0045 \\
\hline 14 & 0 & 0 & 0 & 0 & 0 & .0172 & 0 & .0016 \\
\hline 15 & 0 & 0 & 0 & 0 & 0 & .0098 & 0 & .0004 \\
\hline 16 & 0 & 0 & 0 & 0 & 0 & .0043 & 0 & 0 \\
\hline 17 & 0 & 0 & 0 & 0 & 0 & .0014 & 0 & 0 \\
\hline 18 & 0 & 0 & 0 & 0 & 0 & .0004 & 0 & 0 \\
\hline 19 & 0 & 0 & 0 & 0 & 0 & 0 & 0 & 0 \\
\hline 20 & 0 & 0 & 0 & 0 & 0 & 0 & 0 & 0 \\
\hline
\end{tabular}

summed to get the complex hierarchically modulated signal. In this method, the local content and global contents are

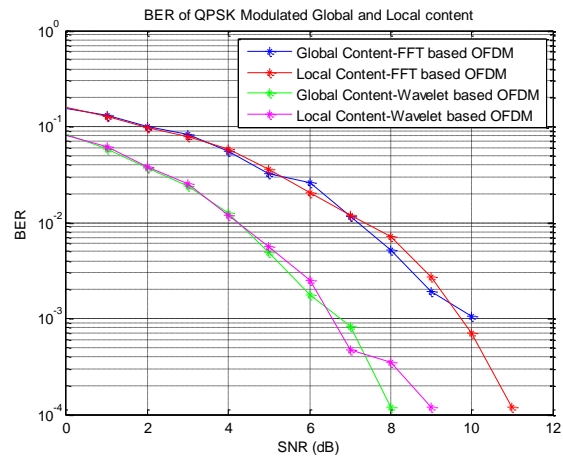

Fig 4.BER of QPSK Modulated global and local content

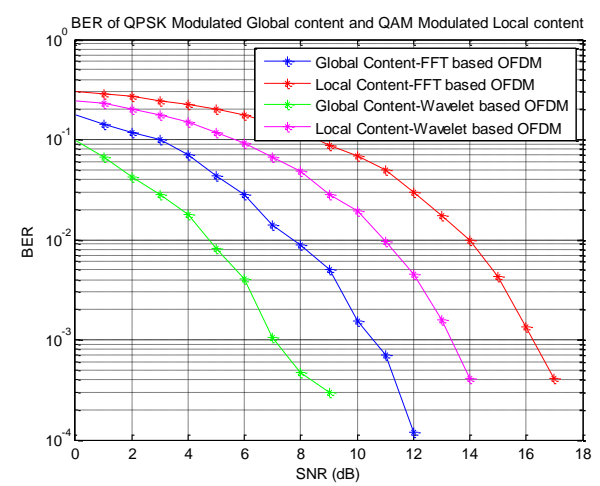

Fig 5.BER of QPSK modulated global content and QAM modulated local content.

\subsection{Performance comparison of Existing and Proposed} work:

Existing work deals with hierarchical modulation where local and global information is separately encoded, modulated and embedded in a single constellation. This is achieved by the selection of appropriate modulation schemes for both the bit stream. In the case of proposed work, the global and the local content are transmitted by selecting the desired subcarrier of the OFDM symbol.

Fourier based system: The Fourier based selective subcarrier method and hierarchical modulation method of transmitting local and global information in a single frequency network are compared. In the analysis shown in Fig 6 and 7, the difference in SNR requirement for both the local and global content is high. For the case, if both global and local content is QPSK modulated, to attain the error rate $10^{-4}$, the global content requires $15 \mathrm{~dB}$ SNR and nearly $22 \mathrm{~dB}$ for local content for the hierarchical modulation technique. This is nearly $5 \mathrm{~dB}$ more for global content and $12 \mathrm{~dB}$ more in local content as compared with selective subcarrier method. This is illustrated in Fig 6.

The analysis done with QPSK modulated global content and QAM modulated local content shown in Fig. 7 also illustrates that a greater requirement of SNR in hierarchical modulated system. The SNR requirement varies by nearly $13 \mathrm{~dB}$ for global content and $7 \mathrm{~dB}$ with local content.. 


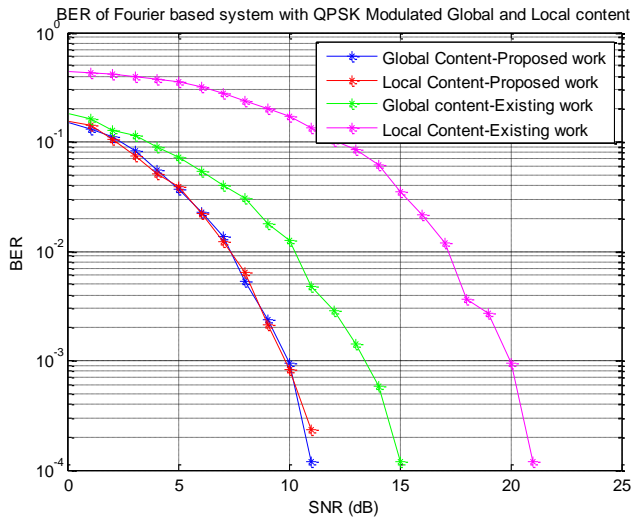

Fig. 6..BER of FFT based system with QPSK modulated global and QPSK modulated local Content

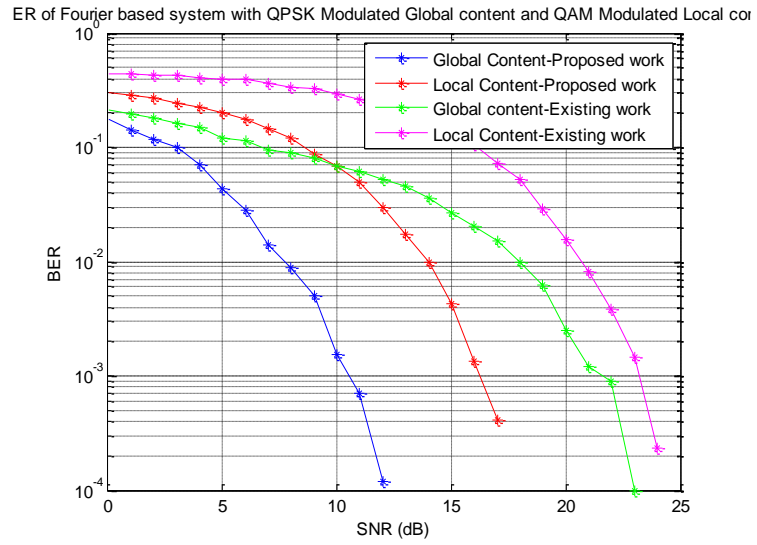

Fig. 7.BER of FFT based system with QPSK modulated global and QAM modulated local Content

Wavelet-based system: Existing and Proposed work with the wavelet-based system is compared in Fig. 8 and 9. In fig. 8 , both the global and local content gives the same error performance in case of the selected subcarrier method. On the other hand, the hierarchical modulated system shows a variation in error performance of both the streams. To attain the error rate $10^{-4}$, the global content requires $12 \mathrm{~dB}$ SNR and nearly $19 \mathrm{~dB}$ for local content. This is nearly $4 \mathrm{~dB}$ more for global content and $11 \mathrm{~dB}$ more in local content as compared with proposed work.

The analysis done with QPSK modulated global content and QAM modulated local content shown in Fig. 9 also explains that a greater requirement of SNR in the hierarchical modulated system. The SNR requirement varies by nearly $10 \mathrm{~dB}$ for global content and $7 \mathrm{~dB}$ with local content.

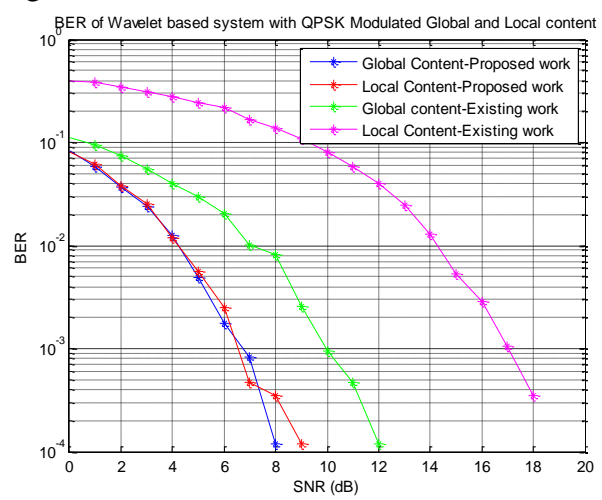

Fig. 8.BER of the wavelet-based system with QPSK modulated global and local content

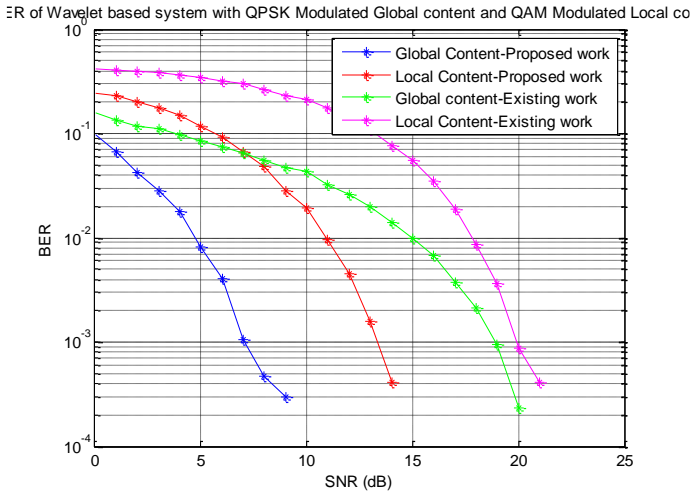

Fig. 9.BER of the wavelet-based system with QPSK modulated Global and QAM modulated local Content

\section{CONCLUSION}

In this paper, the concept of the selected subcarrier method for transmitting the local and global information in a single frequency network has been proposed. Equations are framed to prove the Orthogonality of subcarrier in OFDM symbols. The analysis of the system is done for both Fourier based and wavelet-based OFDM. The analysis proves that the wavelet-based system shows an enhanced performance compared with the Fourier based system. The SNR requirement to attain a specific error rate is less. Also, the performance of the selected subcarrier method is compared with hierarchical modulation based system for both Fourier and wavelet OFDM. In all the case the wavelet outperforms the conventional Fourier based system.

\section{REFERENCES}

1 Nee, R. V., \& Prasad, R. (2000). OFDM for wireless multimedia communications. Artech House, Inc.

2 Hong Jiang; Paul Wilford; Steve Wilkus, "Providing Local Content In A Hybrid Single Frequency Network Using Hierarchical Modulation", IEEE Transactions On Broadcasting, 2010,Vol. 56, No. 4,Pp. 532-540.

3 Jaime Lopez-Sanchez; Jan Zollner, "Technical Solutions for Local Service Insertion in DVB-NGH Single Frequency Networks", IEEE Transactions on Broadcasting, 2014, Vol. 60, No. 2, Pp.293-231.

4 Zoellner J; Robert J, "Local Service Insertion in Terrestrial Single Frequency Networks Based on Hierarchical Modulation", IEEE International Conference on Consumer Electronics, 2012, Pp: 616 617.

5 Hong Jiang; Paul A. Wilford. "A Hierarchical Modulation for Upgrading Digital Broadcast Systems", IEEE Transactions on Broadcasting, 2005, Vol. 51, No. 2, Pp. 223-229.

6 Chandrakala.k, Jayakumari j,"An Efficient Hierarchical Modulation based OFDM transmission scheme for Digital video broadcasting", International Journal of Engineering transaction A:Basics Vol. 31,No 4,(April 2018) Pp 184-191.

7 Meric Hugo; JeromeLacan," Generic Approach for Hierarchical Modulation Performance Analysis: Application to DVB-SH”, Wireless Telecommunications Symposium .2011

8 Chandrakala.k, Jayakumari.J,'Mathematical Modelling and analysis of Hierarchical Modulation in AWGN and Rayleigh channel'.Int.J.Wireless and mobile computing.Vol.16,No.1,Page 72-79.

9 Baig, S., Asif, H. M., Umer, T., Mumtaz, S., Shafiq, M., \& Choi, J. G. (2018). High Data Rate Discrete Wavelet transform-based PLC-VLC Design for $5 \mathrm{G}$ Communication Systems. IEEE Access, 6 52490-52499. 
10 Ceniklioğlu, B., Özen, A., \&Develi, I. (2018, May). Zero-tail discrete wavelet transform spread OFDM system for 5G and beyond. In 2018 26th Signal Processing and Communications Applications Conference (SIU) (pp. 1-4). IEEE.

11 Ghanim, M. F. (2019). Performance investigation of WOFDM for 5G wireless networks. International Journal of Electrical \& Computer Engineering (2088-8708), 9.

12 Dibal, P. Y., Onwuka, E. N., Agajo, J., \&Alenoghena, C. O. (2018). Application of wavelet transforms in spectrum sensing for cognitive radio: A survey. Physical Communication, 28, 45-57.

13 Hamad, N., Takruri, M., \&Barhoush, M. (2019). Wavelet based multicarrier CDMA system. International Journal of Electrical \& Computer Engineering (2088-8708), 9.

14 Hosseini, H., Fisal, N., \& Syed-Yusof, S. K. (2010). Wavelet packet based multicarrier modulation for cognitive UWB systems. Signal Processing: An International Journal (SPIJ), 4(2), 75-84.

15 Dilmaghani, R., \& Ghavami, M. (2007, September). Wavelet vs. Fourier based UWB systems. In 2007 IEEE 18th International Symposium on Personal, Indoor and Mobile Radio Communications (pp. 1-5). IEEE.

16 Dilmaghani, R., \& Ghavami, M. (2008). Comparison between wavelet-based and Fourier-based multicarrier UWB systems. IET Communications, 2(2), 353-358.

17 Goyani, A., \& Shah, R. (2015). A Review-Performance comparison of conventional and wavelet-based OFDM system. International Journal of Innovative Science, Engineering \& Technology, 2(3), 517-520.

18 Zayer, W. H., \&Kateeb, A. (2019, May). OFDM based FFT compared with OFDM based wavelet transform. In IOP Conference Series: Materials Science and Engineering (Vol. 518, No. 5, p. 052011). IOP Publishing.

\section{AUTHORS PROFILE}

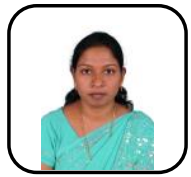

Chandra kala.K received a BE degree in Electronics and communication engineering and ME degree in communication systems from Anna university. Her research interest is in wireless communication, Image processing, and neural networks.

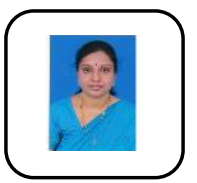

JayaKumari. J received BE degree in Electronics and communication engineering from MS University; Master degree in Applied Electronics and Instrumentation from Kerala University and Ph.D. from Kerala University in 2009.She has teaching experience of 19.5 years, research experience of 12 years and administrative experience of 14 years. She had published several papers in international journals. Her research interest includes Wireless communication and Networking, Signal and image processing, Detection \& Estimation Theory, Spread Spectrum Systems and Error-Correcting Codes. She is a Fellow of Institution of Electronics and Telecommunication Engineers (IETE), Institution of Engineers (India) and council of Engineering and Technology also life member of Indian Society for Technical Education (ISTE), and Senior Member of Institution of Electrical and Electronics Engineers (IEEE).

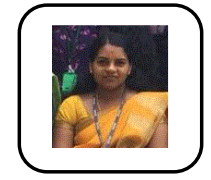

T.K. Sreeja received BE degree in Electronics and Communication Engineering; ME in Communication Systems from Anna University, and $\mathrm{PhD}$ in Electronics and Communication Engineering from Noorul Islam Universityt. She has teaching experience of ten years and research experience of six years. Her research interest includes signal processing and wireless communications. 